\title{
CinÉTICA E EquiLÍBRIO DE BIOSSORÇÃo DE CHUMBo POR MACROALGAS
}

\author{
S. C. S.CALADO ${ }^{1}$, \\ V. L.DA SILVA ${ }^{1^{*}}$ \\ J. Z. DE O. PASSAVANTE ${ }^{2}$; \\ C. A. M DE ABREU ${ }^{1}$, \\ E. S LIMA FILHO ${ }^{3,}$ \\ M. M. M. B. DUARTE ${ }^{4}$ \\ E V. G. S.DINIZ ${ }^{1}$ \\ ${ }^{1}$ Departamento de Engenharia Química, Universidade Federal de \\ Pernambuco-UFPE \\ Rua Tereza Melia s/n, C. Universitária - CEP: 50740-521- Recife-PE, Brasil \\ Telefone (0xx-81)3271-8711 Fax: (0-xx-81)3271-3992-* vlins@npd.ufpe.br \\ ${ }^{2}$ Departamento de Oceanografia, Universidade Federal de Pernambuco \\ ${ }^{3}$ Departamento de Geologia, Universidade Federal de Pernambuco \\ ${ }^{4}$ Fundação Instituto Tecnológico do Estado de Pernambuco - ITEP
}

\section{RESUMO}

Algas coletadas na praia de Jaguaribe, Itamaracá-Pernambuco, selecionadas como Sargassum spp e com a denominação de algas "Arribadas" foram aplicadas em experimentos de equilíbrio e cinética química para se avaliar a capacidade de retenção de $\mathrm{Pb}^{2+}$ em meios líquidos. $\mathrm{O}$ chumbo, enquanto metal muito utilizado em indústrias da região tem os descartes de seus efluentes qualificados como bastantes agressivos ao meio ambiente indicando a necessidade de sua remoção. Os processos de remoção apresentaram retenção máxima do chumbo de 294,1mg/g para Sargassum spp (pH 4) e 138,9mg/g e 95,2 mg/g para algas “Arribadas” (pH 4 e 3) respectivamente. Os experimentos cinéticos realizados demonstraram que $85 \%$ do metal foi retido em 30 minutos e que

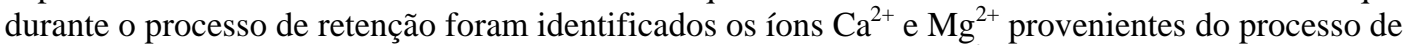
troca iônica com as algas. A quantidade total de íons $\mathrm{H}^{+}$e $\mathrm{Pb}^{2+}$ removidos da solução, foi aproximadamente igual ao total de íons $\mathrm{Ca}^{2+}$ e $\mathrm{Mg}^{2}$ liberados em solução a pH 4. Em pH 3, constatase uma liberação um pouco maior de $\mathrm{Ca}^{2+}$ e $\mathrm{Mg}^{2+}$ em relação ao $\mathrm{Pb}^{2+}$ adsorvido pelos sítios livres do biossorvente, devido a competição com o íon $\mathrm{H}^{+}$. Os tamanhos de partículas estudados (0,149-0,297 e $0,297-0,590$ ) não foram significativos na cinética de biossorção do $\mathrm{Pb}^{2+}$ sobre as constantes de equilíbrio de 14,8 e 30,9 para algas “Arribadas” e Sargassum spp, respectivamente.

Palavras-chave: biossorção, chumbo, algas “Arribadas”, Sargassum spp, troca iônica.

\section{Abstract}

\section{Kinetic and equilibrium of biosorption of the lead by macroalgaes}

“Arribadas" algaes and Sargassum spp. were collected at the Jaguaribe beach-PE, Brazil. Equilibrium experiments and chemistry Kinetic were conducted in order to evaluate the biossorption capacity of $\mathrm{Pb}^{2+}$ by macroalgae and results compared to the values obtained for Sargassum spp. The maximum lead uptake capacitiy was $294.1 \mathrm{mg} / \mathrm{g}$ (pH 4) by Sargassum spp and for "Arribadas" algaes (pH 4 e 3) were $138.9 \mathrm{mg} / \mathrm{g}$ and $95.2 \mathrm{mg} / \mathrm{g}$, respectivamentily. Kinetics 
experiments conducted have demonstrated that $85 \%$ of metal was retained in $30 \mathrm{~min}$. Calcium and magnese were released in higher quantity to studied algaes. The total amounts of lead and hydrogen ions removed from solution were approximately equal to the total amount of calcium and magnese ions released to the solution at $\mathrm{pH} 4$. In $\mathrm{pH} 3$ total amount of calcium and magnese were higher than lead biosorbed due to competion with the hydrogen ion by the free site of the biomass. The sizes of particulas studied (0.149-0.297 and 0.297-0.590) were not significant to kinetic of biosorption of the lead by equilibrium constants of 14.8 and 30.9 respectively to algaes "Arribadas" and Sargassum spp.

KEY-WORDS: biosorption, lead, “Arribadas” algae, Sargassum spp, "Arribadas", ion exchange.

\section{INTRODUÇÃO}

As algas constituem um grupo extremamente diversificado de organismos bastante primitivos e que habitam, predominantemente a água doce e salgada, bem como todos os meios com graus intermediários de salinidade, inclusive o hipersalino (OLIVEIRA et al, 2001).

O litoral brasileiro apresenta vinte e cinco unidades de conservação que abrigam seis diferentes tipos de ecossistemas. A esta lista deverão ser acrescentadas unidades sob jurisdição estadual e municipal. Os ambientes que abrigam as floras mais ricas e diversificadas de algas bênticas são os de costões rochosos e recifes. Em áreas de recifes, como o litoral pernambucano, os gêneros e/ou espécies mais freqüentes ou dominantes são bastante variáveis. Podem ser destacados, na região entre marés, Halimeda spp, Dictyopteris spp, Cryptonemia crenulata, Hypnea musciformis, Osmundaria obtusiloba, Gracilaria spp, Gelidium spp, Sargassum spp, entre muitos outros. Nestas regiões, nos fundos não consolidados é comum o domínio de Halodule wrightii (angiosperma) e Caulerpa spp. Nas regiões permanentemente submersas do infralitoral pode-se destacar Sargassum spp, Halimenia spp, Caulerpa spp, Dictyota spp, Cryptonemia spp, Gracilaria spp, Peyssonnelia spp e Lobophora variegata, dentre outras. Com relação à exploração de espécies para fins comerciais, a atividade de maior porte no país diz respeito à coleta de algas vermelhas dos gêneros Gracilaria e Hypnea na costa nordeste do país, particularmente no trecho que se estende do Ceará até a Paraíba para produção de kapa-carragenano. Outras algas vermelhas são a Porphyra spp, usada como alimento, e a Pterocladiella capilacea, usada para produção de ágar-ágar. Há registro de utilização de Ulva, um gênero de alga verde, e Enteromorpha como adubo na região de Rio Grande (RS), porém em pequena escala (OLIVEIRA et al., loc. cit).

A grande quantidade de algas "Arribadas" nas praias de certas regiões ao longo da costa brasileira podem ser utilizadas como adubo. Muitas vezes são incineradas ou enterradas pelas prefeituras locais devido ao mau cheiro que provocam pela deterioração da matéria orgânica, afastando desta forma os usuários dos ambientes costeiros. WALKER \& KENDRICK (1998) verificaram que a coleta desta biomassa arribada na Austrália foi considerada prejudicial para algumas populações de pássaros e comunidades bentônicas. Por isso, faz-se necessário fazer uma análise do papel ecológico desta biomassa algal nas praias brasileiras. Experimentos realizados no estado da Paraíba constataram que a decomposição destas algas é um fator importante na produtividade da zona costeira. Dentre as algas pardas, o Sargassum spp e a Laminaria spp são pouco exploradas, não havendo registros de impactos significativos nas populações naturais. Em relação às algas verdes, a única eventualmente explorada é a Ulva lactuca de larga distribuição e que está associada a ambientes eutrofizados (OLIVEIRA et al, 2001).

Segundo MATHEICKAL \& YU (1996 e 1999) e VOLESKY \& SHIEWER (1999), as algas constituem biossorventes de baixo custo com grande potencial para serem aplicadas em estudos de biossorção. Contudo, faz-se necessário entender o papel destes organismos na natureza, 
avaliando sua importância ecológica e verificando o impacto que sua utilização poderia provocar no meio ambiente.

Algumas algas têm demonstrado grande capacidade de retenção de metais especialmente as algas pardas. O Sargassum spp, que é um gênero das algas pardas, têm sido estudado como um dos melhores biossorventes devido a sua alta capacidade de retenção, resistência química e mecânica de metais pesados e radioativos como o urânio, além de uma melhor resistência ao ataque ácido, resultando em menor perda de massa quando em contato com correntes de efluentes líquidos(YANG \& VOLESKY, $1999^{\mathrm{a}}$ e 1999 ${ }^{\mathrm{b}}$; FIGUEIRA et al, 2000).

Por isso, este gênero de alga foi escolhido para comparação com as algas "Arribadas”. Íons de metais pesados representam um risco para a vida animal e vegetal mesmo quando presente em baixas concentrações. Eles se acumulam através da cadeia alimentar e podem ser bastante prejudiciais para a vida humana. Além do mais, o esgotamento dos recursos naturais torna a recuperação destes elementos algo muito importante. O chumbo é considerado como alta prioridade devido ao risco ambiental e taxa de esgotamento das reservas naturais. As atuais tecnologias para remoção de metais pesados de efluentes industriais são de alto custo, produzindo lamas tóxicas criando desta maneira um problema secundário. A biossorção representa uma alternativa para o uso deste material no tratamento de efluentes industriais. As indústrias têm interesse na aplicação deste material para a remoção de metais pesados de seus resíduos industriais, por ser uma alternativa de baixo custo, economicamente competitiva e baixo potencial de agressão ao meio ambiente.(KRATOCHVIL e VOLESKY,1998 ${ }^{\mathrm{a}}$ e 1998 ; FIGUEIRA et al.,2000).

O presente trabalho tem como objetivo estudar os mecanismos envolvidos no processo de adsorção do chumbo pelas algas através de equilíbrio e cinética química, de modo a estabelecer a quantidade de íons $\mathrm{Pb}^{2+}$ retidos, visando tornar esta técnica mais eficientemente otimizada e aplicada.

\section{MATeriais e MÉtodos}

\section{Materiais}

Foram utilizados no presente desenvolvimento os seguintes reagentes de grau analítico: nitrato de chumbo (SIGMA) e ácido nítrico 65\% (MERCK). A água utilizada nos experimentos foi

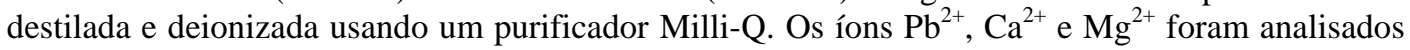
por espectrometria de emissão por Plasma Indutivamente Acoplado (ICP-AES, Iris-Termo Jarrel Ash Coorporation). A concentração hidrogeniônica foi determinada via potenciometria utilizando um pHmetro da Radelkis OP208/1 e um eletrodo combinado de vidro da Radelkis OP-0808-P.

\section{Métodos}

\section{Preparação do biossorvente}

As algas "Arribadas" e Sargassum spp foram coletados na praia de Jaguaribe, Itamaracá, Pernambuco-Brasil (Figura 1), lavadas com água potável, secas em Casa de Vegetação a temperatura de $37 \pm 2^{\circ} \mathrm{C}$, moídas em moinho de faca com lâminas inoxidáveis (Tecnal) e peneiradas nos tamanhos de partículas de 0,149-0,297mm e 0,297-0,590mm. Depois foram lavadas várias vezes com água destilada até $\mathrm{pH}$ 6,5 e finalmente com água deionizada, e secas em estufa revestida

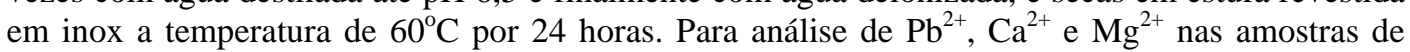
algas seguiu-se a metodologia da AOAC digerindo-as com $\mathrm{HNO}_{3}$ concentrado. Todos os experimentos foram realizados a temperatura ambiente $\left(27 \pm 2^{\circ} \mathrm{C}\right)$. 


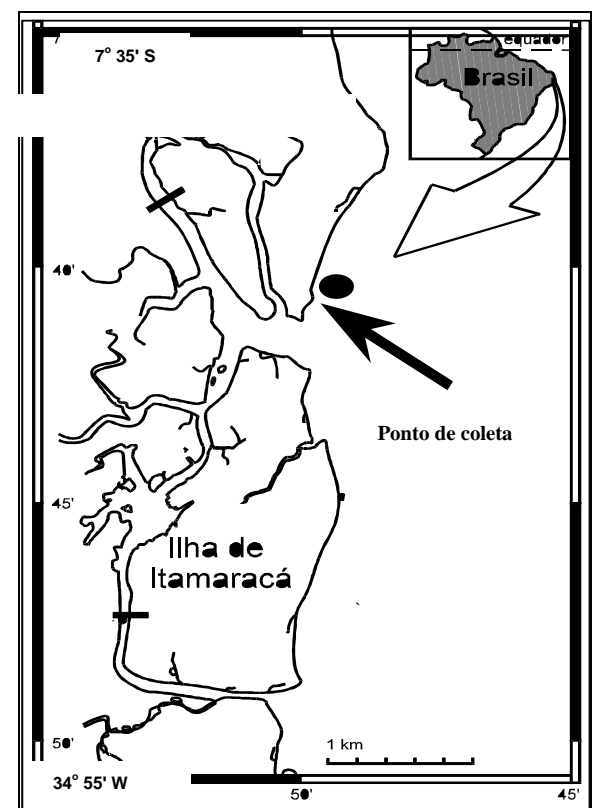

Figura 1 - Mapa da praia de Jaguaribe, Itamaracá - Pernambuco Brasil

\section{Variação de pH durante a biossorção em algas “Arribadas” e Sargassum spp.}

Cerca de 0,1 a $1 \mathrm{~g}$ da biomassa moída e peneirada em tamanho de partículas, 0,1490,297mm foram colocadas em contato com $50 \mathrm{~mL}$ de solução nítrica (pH 1 a 4) contendo 30mg/L de chumbo e o pH foi monitorado até 90 minutos, para avaliar a sua variação durante o processo de retenção do metal.

\section{Experimentos cinéticos de biossorção}

Na preparação dos experimentos cinéticos, $120 \mathrm{mg}$ de nitrato de chumbo foram pesados e dissolvidos em 4 litros de água destilada e deionizada, adicionando-se a seguir $8 \mathrm{~g}$ de biomassa. A solução foi constantemente agitada a uma velocidade de 300 rpm durante 90 minutos em pH inicial 3 e 4 para algas “Arribadas” e pH 4 para algas Sargassum spp.

$\mathrm{O}$ pH foi constantemente controlado pela adição de solução de ácido nítrico 0,1 M. Em tempos que variaram de 30 segundos a 90 minutos, cerca de $10 \mathrm{~mL}$ da solução foi filtrada em papel de filtro quantitativo do tipo Whatman n.542 e o teor de $\mathrm{Pb}^{2+}, \mathrm{Ca}^{2+}, \mathrm{Mg}^{2+}$ e $\mathrm{H}^{+}$do filtrado foi analisado por ICP-AES e potenciometria.

\section{Experimentos de equilíbrio de biossorção}

Um total de 0,1 g de biomassa com tamanho de partícula de 0,297-0,590mm foi colocado em 50mL de solução contendo $30 \mathrm{mg}$ a $800 \mathrm{mg}$ de chumbo em béquer de $100 \mathrm{~mL}$, com agitação 
magnética por 90 minutos, sendo a solução após este período, filtrada em papel Wathman n.542. O $\mathrm{pH}$ foi constantemente controlado para pH 3 e 4 com adição de solução de ácido nítrico $0,1 \mathrm{M}$. As amostras das soluções iniciais e dos filtrados após equilíbrio foram analisadas para se avaliar o teor de chumbo em solução. Os experimentos foram realizados em duplicata.

\section{Determinação da retenção do metal}

A retenção do chumbo pela biomassa foi calculada segundo a equação de balanço a seguir:

$$
\mathrm{q}=\left([\mathrm{M}]_{\mathrm{i}}-[\mathrm{M}]_{\mathrm{f})} \mathrm{V} / \mathrm{m}\right.
$$

na qual, $[\mathrm{M}]_{\mathrm{i}} \mathrm{e}[\mathrm{M}]_{\mathrm{f}}$ são as concentrações inicial e final de chumbo respectivamente, $\mathrm{V}$ é o volume de solução e m é a massa de alga.

A quantidade de próton retida $\Delta \mathrm{Hq}$ foi calculada como sendo a diferença entre a quantidade de prótons adicionada $\left([\mathrm{H}]_{\mathrm{ad}} \mathrm{V}_{\mathrm{ad}}\right)$ para ajuste do $\mathrm{pH}$ e os prótons acumulados em solução $\left(\left([\mathrm{H}]_{\mathrm{f}}-[\mathrm{H}]_{\mathrm{i})} \mathrm{V}\right)\right.$ assim expressa:

$$
\Delta \mathrm{Hq}=\left\{[\mathrm{H}]_{\mathrm{ad}} \mathrm{V}_{\mathrm{ad}}-\left([\mathrm{H}]_{\mathrm{f}}-[\mathrm{H}]_{\mathrm{i}}\right) \mathrm{V}\right\} / \mathrm{m}
$$

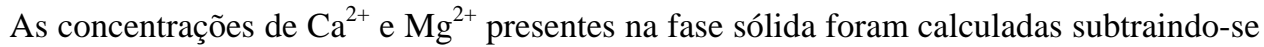
a quantidade liberada de íon na fase líquida do teor total destes íons nos sólidos.

\section{RESUlTADOS EXPERIMENTAIS E DISCUSSÕES}

O metal em solução na sua forma iônica, bem como prótons, são adsorvidos nas superfícies da biomassa algal, onde em contato com as estruturas químicas possuidoras de conteúdos predominantes de cálcio, magnésio e hidrogênio procedem trocas iônicas. Efeitos cinéticos na direção de equilíbrios de adsorção e/ou troca iônica podem ser avaliados, observadas, na fase líquida, as evoluções das concentrações dos componentes citados.

\section{Variação do pH durante a biossorção em algas "Arribadas" e Sargassum spp.}

A elevação do $\mathrm{pH}$ no processo de adsorção do metal em presença de algas "Arribadas" ocorre devido à retenção destes e de prótons em solução pela biomassa e a conseqüente liberação de metais alcalinos para a solução. O processo de troca iônica foi evidenciado pela avaliação cinética do $\mathrm{pH}$ (Figura 2), com o monitoramento deste por um período de 90 minutos, iniciando-se com solução tendo valores de $\mathrm{pH}$ 1, 2, 3 e 4. Nos quatro casos foram observadas bruscas elevações do $\mathrm{pH}$ nos primeiros 5 minutos, devido a uma rápida troca iônica cujo o equilíbrio é atingido em cerca de 10 minutos com valores de $\mathrm{pH}$ entre 7,5 e 7,8 para $\mathrm{pH} 3$ e 4, respectivamente. Para $\mathrm{pH} 2$ inicial, obteve-se equilíbrio com um valor de $\mathrm{pH}$ final de 3,2. Observou-se que a adsorção de chumbo é mais elevada quando há menores teores dos íons $\mathrm{H}^{+}$presentes na solução inicial. Os resultados indicam que o controle do $\mathrm{pH}$ faz-se necessário durante o processo de biossorção para valor de $\mathrm{pH}$ inicial acima de 2, mesmo com pequenas quantidades de biossorvente.

Efeitos de competição entre os íons de $\mathrm{Pb}^{2+}$ e de $\mathrm{H}^{+}$em solução foram avaliados procedendo-se o monitoramento do $\mathrm{pH}$ de soluções iniciais com $\mathrm{pH} 3$ contendo diferentes teores de chumbo. 


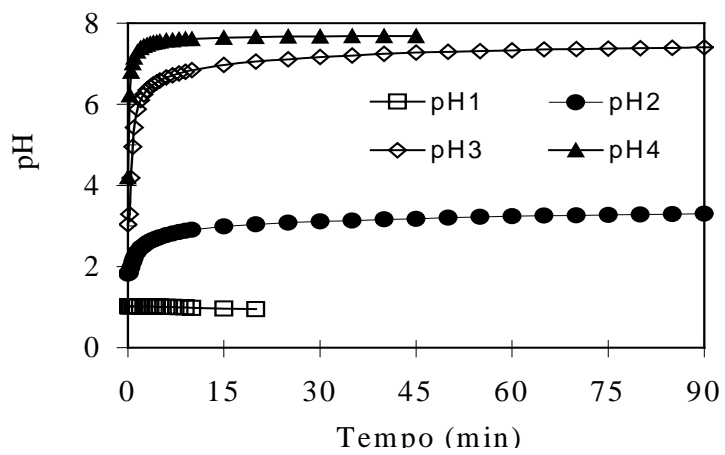

Figura 2 - Variação do $\mathrm{pH}$ a diferentes valores iniciais $(1,2,3,4)$ usando $1 \mathrm{~g}$ de algas "Arribadas".

Na Figura 3, mostra-se que a presença de chumbo reduziu o $\mathrm{pH}$ da fase líquida, indicando a ocorrência de uma competição na adsorção/troca iônica entre os íons $\mathrm{H}^{+}$e $\mathrm{Pb}^{2+}$ pelos sítios da alga. Para soluções contendo biomassa de $0,1 \mathrm{~g}$, o pH final da solução mudou de 5,5 (solução isenta de chumbo) para 5,0 (solução contendo chumbo) representando uma redução correspondente a $0,035 \mathrm{mEq} / \mathrm{g}$ em termos de concentração de íons $\mathrm{H}^{+}$. Maiores quantidades de biomassa (1g) representam um maior número de sítios de ligação que promovem uma maior retenção. Quando se usou 1 g de biomassa, partindo-se de $\mathrm{pH} 3$, o pH final atingiu respectivamente 7,4 e 7,0 para solução isenta de chumbo e contendo chumbo, respectivamente. Experimentos de biossorção de chumbo e algas "Arribadas" foram realizados com massa do metal de 0,1 e 0,5g, visando a quantificação do efeito deste sobre o processo de adsorção/troca iônica alga/metal. Os resultados contidos na Figura 3 mostram um efeito não significativo da redução de $\mathrm{pH}$ em função do tempo para as duas respectivas massas de $\mathrm{Pb}^{2+}: 0,5$ e $1,0 \mathrm{~g}$.

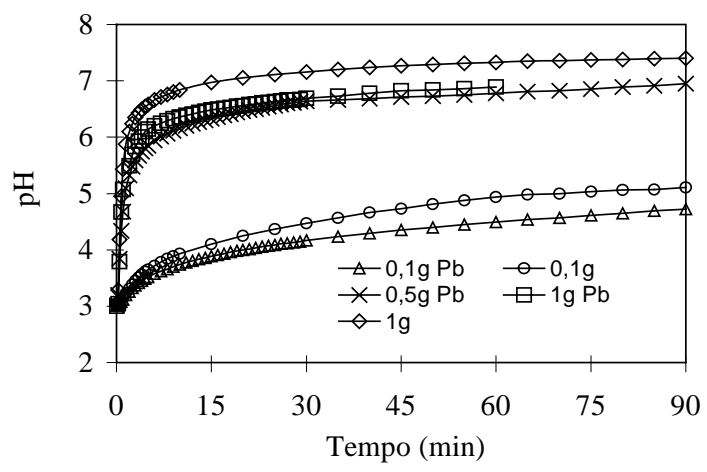

Figura 3 - Variação do pH usando algas "Arribadas" iniciando a $\mathrm{pH} 3$ para diferentes quantidades de biomassa e presença ou não de chumbo em solução.

Experimentos cinéticos com diferentes granulometrias da biomassa algal foram executados para se determinar o menor tempo requerido para que o equilíbrio de retenção fosse atingido, prevendo-se redução da biossorção do $\mathrm{Pb}^{2+}$ por transferência de massa. 
Na Figura 4, para algas “Arribadas”, a pH 4 inicial, estão representados os efeitos do tamanho de partícula entre 0,149-0,297mm e 0,297-0,590mm. Tamanhos inferiores a estes estudados de biossorventes poderiam conduzir a obtenção mais rápida das condições de equilíbrio, em decorrência possivelmente de menores limitações aos efeitos de transferência de massa.

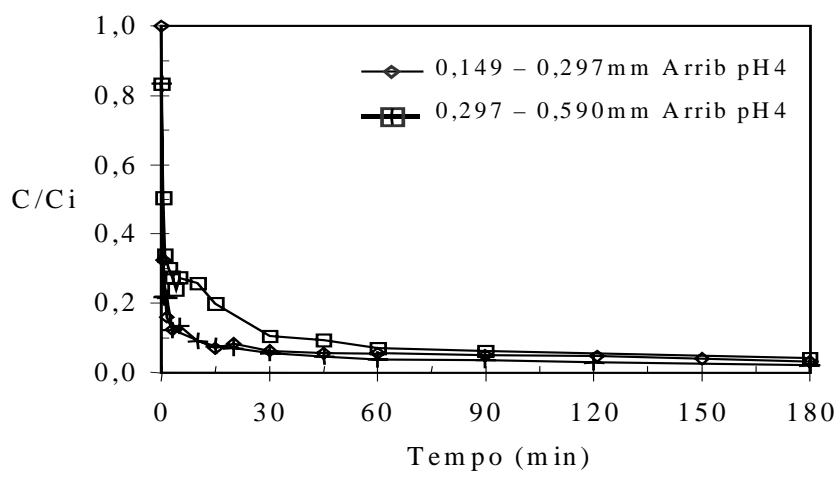

Figura 4 - Efeito do tamanho de partícula na cinética da solução de $\mathrm{Pb}^{2+}$ (30 ppm) usando algas "Arribadas" a pH 4.

Na Figura 5, foram comparadas as algas “Arribadas” e Sargassum spp com a mesma granulometria a $\mathrm{pH} 4$ inicial. A alga Sargassum spp mostrou cinética mais rápida e melhor capacidade de retenção, reduzindo quase totalmente a concentração de chumbo da fase líquida, alcançando retenção total do metal em 30 minutos.

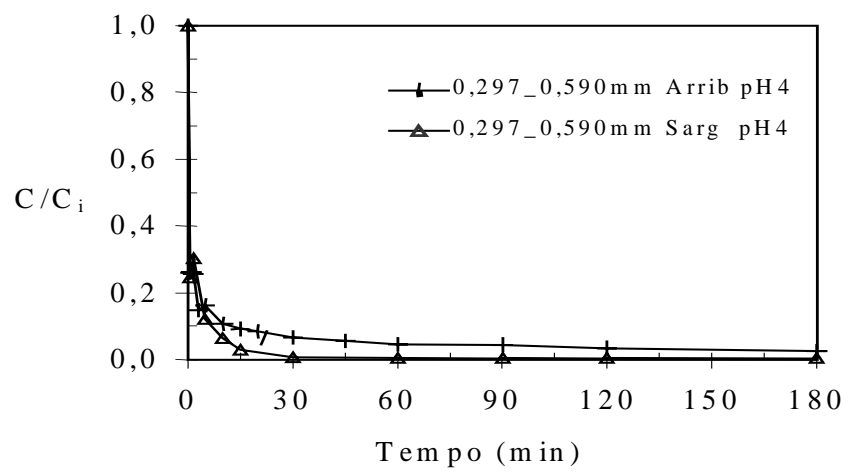

Figura 5 - Efeito da biomassa de algas "Arribadas" e Sargassum spp, na cinética da solução de $\mathrm{Pb}^{2+}(30 \mathrm{ppm})$ a $\mathrm{pH} 4$.

\section{Isotermas de equilíbrio de biossorção}

As isotermas de equilíbrio de retenção de chumbo com as algas “Arribadas” e Sargassum spp em meios de pH de 3 e 4 estão representadas na Figura 6.

Tropical Oceanography, Recife: v. 31, n. 1, p. 27-36, 2003 


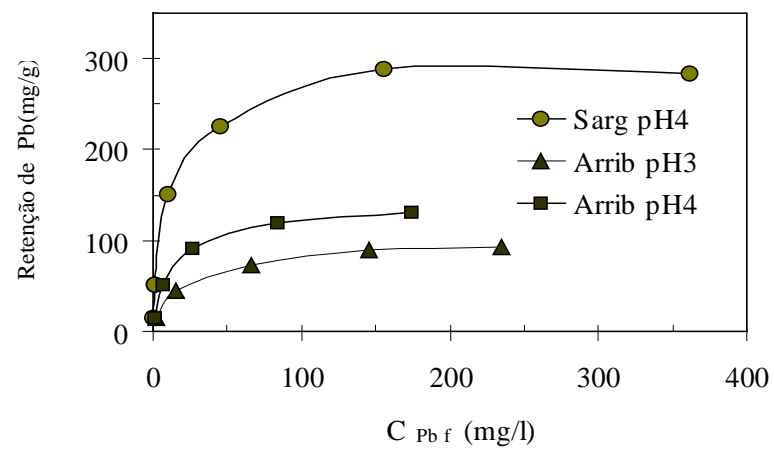

Figura 6 - Curvas das isotermas de retenção de chumbo para as algas “Arribadas” ( $\mathrm{pH} 3$ e 4) e Sargassum spp (pH 4)

Destas avaliações resultaram as capacidades máximas de retenção do chumbo que atingiram 138,9mg/g e 95,2mg/g para algas "Arribadas" a $\mathrm{pH} 4$ e 3, respectivamente e para o Sargassum spp foi de 294,1mg/g a pH 4. Como as algas "Arribadas" são uma mistura de espécies, as quais incluem as verdes, vermelhas e marrons, há diferentes grupos funcionais presentes na biomassa, representando os sítios de troca iônica. MATHEICKAL \& YU (1999) estudando as algas pré-tratadas Durvilae potatorum e Ecklonia radiata obtiveram capacidades de retenção de $1,47 \mathrm{mmol} / \mathrm{g}$ e $1,17 \mathrm{mmol} / \mathrm{g}$ respectivamente para um mesmo $\mathrm{pH}$ (4), valores estes comparáveis aos obtidos para o Sargassum spp a pH 4 (1,42mmol/g).

\section{Troca iônica total}

Os balanços de massa dos íons em decorrência das trocas iônicas com as algas "Arribadas" e Sargassum spp foram realizados considerando as experiências a pH 4 cujas cinéticas em direção do equilíbrio foram mais rápidas. O total de íons liberados para a fase líquida foi aproximadamente igual ao total de íons removidos da solução (Figuras 7 e 8). Os íons $\mathrm{Ca}^{2+}$ e $\mathrm{Mg}^{2+}$ foram trocados por $\mathrm{Pb}^{2+} \mathrm{e} \mathrm{H}^{+}$. As quantidades máximas de $\mathrm{Ca}^{2+}$ liberado em solução foram de 2,88mEq/g e 1,30mEq/g, enquanto que as quantidades máximas de $\mathrm{Mg}^{2+}$ liberado em solução foram de 0,27 mEq/g e 0,20mEq/g, para as algas Sargassum spp e algas " Arribadas" respectivamente.

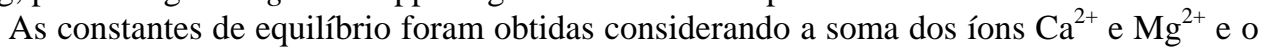
total de íons liberados no sistema. A partir das evidências experimentais, uma função objetivo $\mathrm{Fo}=\Sigma\left\{\left(\left[\mathrm{Pb}^{2+}\right]_{\text {real }}\left[\mathrm{Pb}^{2+}\right]_{\text {calc }}\right) /\left[\mathrm{Pb}^{2+}\right]_{\text {real }}\right\}^{2}$, sendo $\left[\mathrm{Pb}^{2+}\right]_{\text {real }}$ a concentração medida e $\left[\mathrm{Pb}^{2+}\right]_{\text {calc a }}$ concentração calculada, foi minimizada, comparando-se os valores das concentrações de chumbo calculadas via constante de equilíbrio e aquelas determinadas experimentalmente. As constantes de equilíbrio assumiram valores de 14,8 e 30,9 para as algas "Arribadas" e Sargassum spp, respectivamente, denotando a ocorrência de biossorção favorável nos dois casos. Os erros experimentais calculados para a retenção de chumbo nos experimentos de equilíbrio foram de 2,4mg/g e 2,0mg/g para as algas “Arribadas", a pH 3 e 4, respectivamente, enquanto para o Sargassum spp foi de 6,9mg/g obtido a pH 4,0. 


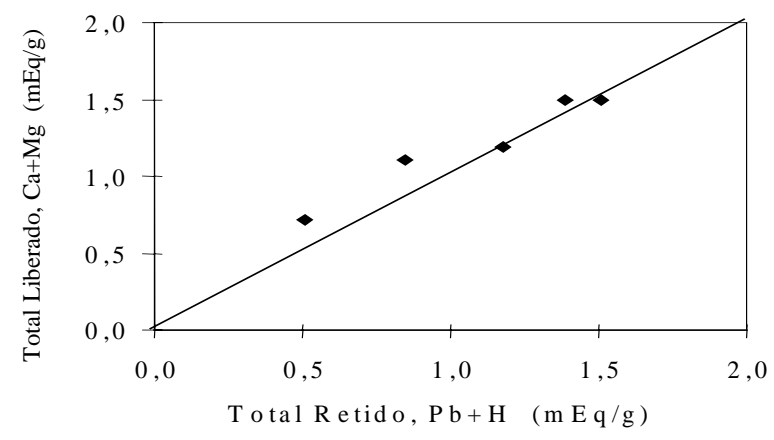

Figura 7 - Troca iônica obtida pela isoterma das algas "Arribadas" a pH 4, considerando a liberação de $\mathrm{Ca}^{2+} \mathrm{e}$ $\mathrm{Mg}^{2+}$, e a remoção de $\mathrm{H}^{+}$e $\mathrm{Pb}^{2+}$.

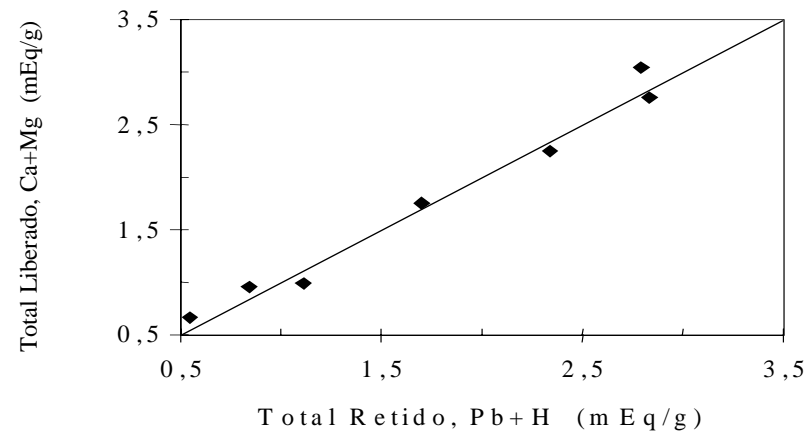

Figura 8 - Troca iônica obtida pela isoterma das algas Sargassum spp a pH 4, considerando a liberação de $\mathrm{Ca}^{2+}$ e $\mathrm{Mg}^{2+}$, e a remoção $\mathrm{H}^{+}$e $\mathrm{Pb}^{2+}$.

\section{CONCLUSÕES}

Os resultados obtidos mostraram que as algas "Arribadas" possuem metade da capacidade de retenção do $\mathrm{Pb}^{2+}$ relativamente àquela obtida para o Sargassum spp a pH 4. Em pH 3,0 há menor troca com o $\mathrm{Pb}^{2+}$ devido a uma maior competitividade deste metal com o próton pelos sítios de ligação do biossorvente.

A capacidade máxima de remoção do íon $\mathrm{Pb}^{2+}$ foi de 294,1 mg/g (pH 4) pelo Sargassum spp e para algas “Arribadas” foram 138,9 mg/g e 95,2 mg/g, respectivamente para pH 4 e 3.

Os experimentos cinéticos realizados demonstraram que $85 \%$ do metal foi retido em 30 minutos e que durante o processo de retenção foram identificados os íons $\mathrm{Ca}^{2+}$ e $\mathrm{Mg}^{2+}$ provenientes

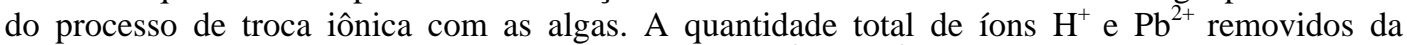
solução, foi aproximadamente igual ao total de íons $\mathrm{Ca}^{2+}$ e $\mathrm{Mg}^{2}$ liberados em solução a $\mathrm{pH} 4$.. Em pH 3, constata-se uma liberação um pouco maior de $\mathrm{Ca}^{2+}$ e $\mathrm{Mg}^{2+}$ em relação ao $\mathrm{Pb}^{2+}$ adsorvido pelos sítios livres do biossorvente, devido a competição com o íon $\mathrm{H}^{+}$. Os tamanhos de partículas 
estudados $\left(0,149-0,297\right.$ e 0,297-0,590) não foram significativos na cinética de biossorção do $\mathrm{Pb}^{2+}$ sobre as constantes de equilíbrio de 14,8 e 30,9 para algas “Arribadas” e Sargassum spp, respectivamente.

Considerando a disponibilidade da biomassa algal e visando a remoção de teores de chumbo de efluentes aquosos, mostrou-se a possibilidade de aplicação de algas “Arribadas” e Sargassum spp no tratamento de rejeitos industriais.

\section{REFERÊNCIAS BIBLIOGRÁFICAS}

FIGUEIRA, M. M.; VOLESKY, CIMINELLI, B. V. S. T.; RODDICK, F. A. Biosorption of Metals in Brown Seaweed Biomass. Wat. Res., v. 34, n. 1, p. 196-204. 2000

KRATOCHVIL, D; VOLESKY, B. Biosorption of Cu from Ferruginous Wastewater by Algal Biomass. Wat. Res. v. 32, n. 9 p. 2760-2768. 1998a

KRATOCHVIL, D.; VOLESKY, B. Reviews: Advances in the biosorption of heavy metals. Tibtech, Elsevier Science, v. 16, p. 291-300. 1998b.

MATHEICKAL, J. T.; YU, Q. Biosorption of lead from aqueous solutions by Marine algae Ecklonia radiata. Wat. Sci. and Technol., v. 34, n. 9, p. 1-7. 1996.

MATHEICKAL, J. T.; YU, Q. Biosorption of lead (II) and copper(II) from aqueous solutions by pre-treated biomass of Australian marine algae. Bio. Techn., v. 69, p. 223-229. 1999,

OLIVEIRA, E. C. HORTA, P. A, AMANCIO, C. E.; SANTANNA, C. L. - Algas e Angiospermas marinhas bênticas do litoral brasileira. Instituto de Biocências da Universidade de São Paulo. Instituto de Botânica, Seção de Ficologia. Disponível em: http://www.

bdt.org.br/workshop/costa/algas Acesso em: 31 dez. 2001.

VOLESKY, B.; SHIEWER, S. Encyclopedia of Bioprocess Technology. M. C. Flickinger and S.W. Drew, John Wiley \& Sons Inc., 1999, 439p.

YANG, J.; VOLESKY, B. Biosorption of Uranium on Sargassum biomass. Wat. Res, v. 33, n. 15, p. 3357-3363. 1999a.

YANG, J.; VOLESKY, B. Cadmium biosorption rate in protonated Sargassum biomass. Environ. Sci. Technol.,v. 33, p. 751-757. 1999 b.

WALKER, D. I.; KENDRICK, G. A. Threats to macroalgal diversity: marine habitat destruction and fragmentation, pollution and introduced species. Botanic Marine, v. 41, p. 105-112. 1998. 